\title{
Resección de aneurisma de arteria esplénica por vía laparoscópica conservando el bazo*
}

\author{
Drs. MARCO A. RIVERA A. ${ }^{1,2}$, JUAN I. BRAVO F. ${ }^{1}$, CARLO U. MARINO C. ${ }^{2}$, JUAN C. TOBAR M. ${ }^{1}$ \\ 1 Servicio de Cirugía Hospital Base, Curicó. \\ 2 Escuela de Medicina Universidad Católica del Maule, Talca. \\ Chile.
}

\begin{abstract}
Laparoscopic surgical excision of a splenic aneurism

Elective surgical repair is indicated for splenic aneurisms when they are wider than $20 \mathrm{~mm}$, during pregnancy and when a progressive increase in diameter is documented. We report a 63 years old female with a history of hypertension, presenting with pain in the right upper quadrant. An abdominal CAT scan disclosed a hepatic cyst and an aneurism of the splenic artery of $20 \mathrm{~mm}$ diameter. A laparoscopic excision of the aneurism was carried out without performing a splenectomy. A CAT scan done one month later postoperative period detected a small lateral spleen infarction and a subcapsular collection of $2 \mathrm{~cm}$ diameter.
\end{abstract}

Key words: Esplenic aneurism, laparoscopic surgery, spleen.

\section{Resumen}

Los aneurismas esplénicos, a pesar de ser los aneurismas viscerales más frecuentes, son inhabituales. Su resolución quirúrgica electiva se considera cuando presentan un diámetro mayor a 2 centímetros, son sintomáticos, se presentan en pacientes embarazadas o se demuestra su crecimiento progresivo. Ello debido a que las variables mencionadas aumentan su riesgo de ruptura. El manejo endovascular mediante embolización de la arteria esplénica, o la cirugía abierta con aneurismectomía con esplenectomía u, ocasionalmente, sólo con reparación arterial, corresponden a los manejos electivos más aceptados. Se presenta un caso clínico de aneurisma de arteria esplénica, manejado electivamente con una técnica quirúrgica novedosa. Se trata de una paciente en quien, por vía laparoscópica, se realizó resección del aneurisma y se preservó el bazo. La presentación de este caso, con un abordaje menos invasivo y que permite evitar la esplenectomía, nos invita a replantear los manejos clásicos endovasculares o abiertos, que son más complejos y, habitualmente, requieren la esplenectomía.

Palabras clave: Aneurisma arteria esplénica, aneurismectomía laparoscópica, aneurisma visceral.

*Recibido el 14 de septiembre de 2010 y aceptado para publicación el 19 de diciembre de 2010.

Correspondencia: Dr. Marco A. Rivera A.

Avda. San Miguel 3605, Casilla 617, Talca, Chile. Fax: 5671203435

Marcoariv@yahoo.com 


\section{Introducción}

Los aneurismas de la Arteria Esplénica (AAE) son los aneurismas viscerales más comunes $(60 \%)^{1}$. Por lo general son asintomáticos, afectan en una mayor proporción a mujeres ${ }^{2}$ y la edad promedio al momento del diagnóstico es de 52 años. 22\% de los pacientes tiene aneurismas múltiples³. La etiología más común es la fibrodisplasia y la hipertensión portal ${ }^{4}$.

Su ruptura $(<2 \%$ de los casos) tiene una mortalidad que alcanza al $36 \% 5$, lo que habla de la importancia de conocer la presencia de este tipo de aneurismas.

El gold standard para el diagnóstico de aneurismas esplénicos es la angiografía ${ }^{6}$ que permite localizar y definir el tamaño de éstos. El manejo quirúrgico se plantea en: aneurismas cuyo diámetro supera los 2 centímetros, sintomatología relacionada a la presencia de éste, aneurismas en pacientes embarazadas, o cuando se demuestra un crecimiento de éste ${ }^{7}$. Aquellos aneurismas cuyo diámetro oscila entre 1 y 2 centímetros deben estudiarse con imágenes cada 6 meses.

La resolución quirúrgica de los AAE puede ser por medio de cirugía abierta que tiene eficiencia y durabilidad bien documentada ${ }^{8}$, cirugía laparoscópi$\mathrm{ca}^{9-12} \mathrm{o}$ terapia endovascular ${ }^{13,14}$.

Presentamos un caso donde se escogió la resolución por vía laparoscópica, obteniendo resultados favorables tanto en el intraoperatorio, como en la evolución postoperatoria de nuestra paciente.

\section{Caso clínico}

Paciente de sexo femenino, 63 años de edad, con antecedentes de hipertensión arterial en tratamiento farmacológico.

Por cuadro de molestias en hipocondrio derecho de 1 año de evolución se realizó una ecotomografía abdominal, identificándose un quiste hepático complejo de 22 x $24 \mathrm{~mm}$. Para complementar el estudio, se realizó TC abdominal, que confirmó quiste hepático simple y, como hallazgo, informó la presencia de una imagen adyacente al hilio esplénico, de 20 mm de diámetro, sugerente de aneurisma de arteria esplénica (Figura 1).

El examen físico no presentó hallazgos importantes.

Dado el diámetro del aneurisma se decide su resolución quirúrgica electiva, mediante un abordaje laparoscópico.

Con la paciente en decúbito lateral derecho en $45^{\circ}$, se realizó un acceso con 4 puertos (2 de $10 \mathrm{~mm}$ y 2 de $5 \mathrm{~mm}$ ). Sección del ligamento esplenocólico y decolamiento de la cola del páncreas para abordar la parte inferior del hilio esplénico donde se encontraba el aneurisma. En el transcurso de la disección se usó Ligasure®. Expuesta la primera bifurcación de la arteria esplénica y su segmento más proximal, se identifica el aneurisma fusiforme. El control vascular proximal y distal, se realizó mediante doble bloqueo con clips poliméricos (Hem-o-lock $®)$. Finalmente, se secciona la arteria esplénica y se extrae, el aneurisma, por el $3^{\circ}$ puerto. Cierre de los puertos de $10 \mathrm{~mm}$ con Vicryl ${ }^{\circledR}$ $1-0$, el drenaje fue exteriorizado por el $4^{\circ}$ puerto y retirado al $2^{\circ}$ día. Su evolución postoperatoria no presentó complicaciones. Fue dada de alta al tercer día.

La paciente fue controlada al mes, encontrándose asintomática, la TC de control demostró bazo de 12,6 x 8,9 cm, con imagen de pequeño infarto lateral y el resto con impregnación normal. Pequeña colección de $2 \mathrm{~cm}$ de diámetro, subcapsular (Figura 2).

Nueve meses más tarde, recibió el alta médica.

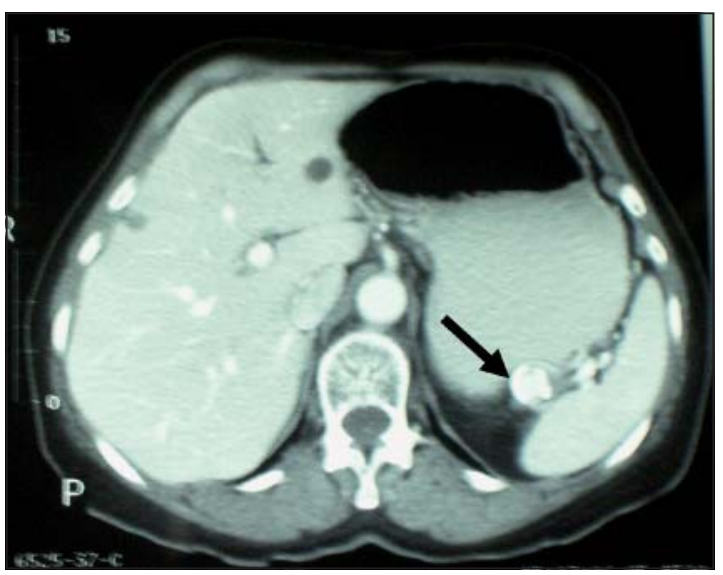

Figura 1. Imagen tomográfica contrastada en una mujer de 63 años, demostrando un aneurisma esplénico de 2,0 cm de diámetro. Flecha: Aneurisma pre-hiliar.

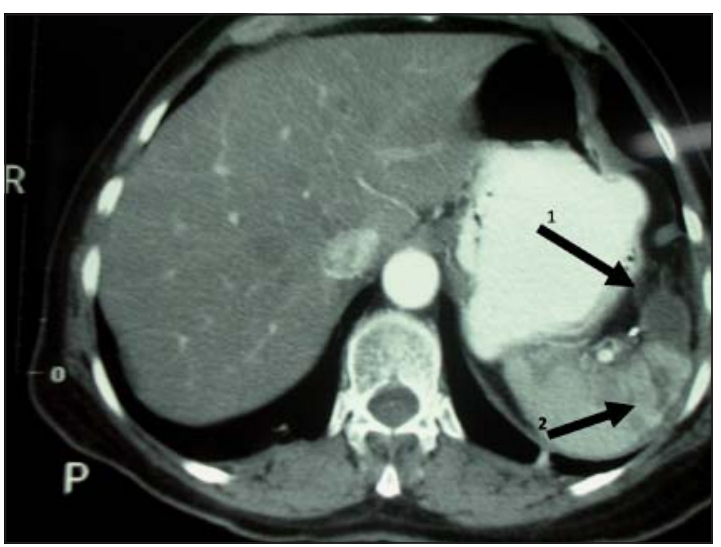

Figura 2. Imagen tomográfica contrastada de una paciente femenina de 63 años al mes de someterse a una aneurismectomía laparóscopica. Flecha 1: Colección hiliar para-esplénica. Flecha 2: Pequeño infarto subcapsular. 


\section{Discusión}

La resección electiva de los aneurismas esplénicos conlleva un riesgo de complicaciones mayores, incluyendo una mortalidad que puede alcanzar a $25 \%$ en casos de aneurismas rotos ${ }^{5}$. Además, las técnicas más habituales de ligadura de la arteria esplénica o la resección del aneurisma con reparación de la arteria en forma término-terminal, conllevan un riesgo de esplenectomía como consecuencia de la isquemia distal del órgano.

La introducción de las técnicas endovasculares y su masificación, permitió también, enfrentar a los aneurismas esplénicos mediante estas técnicas. Así, en la actualidad, puede proponerse para el tratamiento de un aneurisma esplénico la embolización con coils o metacrilato, u ocasionalmente, el aislamiento del aneurisma con el implante de un stent cubierto.

Sin embargo, con estas técnicas, el requerimiento de esplenectomía persiste y se han reportado isquemias del bazo postprocedimiento que alcanzan hasta un $29 \%{ }^{15}$. Por otro lado, en el manejo endovascular de estos aneurismas se ha reportado tasas de complicaciones mayores hasta de un $36,4 \%{ }^{16}$.

Este caso presentado constituye, hasta donde conocemos, el primer reporte de un caso exitoso con abordaje laparoscópico que se haya hecho en nuestro país y nos invita a aunar nuestras experiencias para replantearnos los abordajes de elección o incorporar una opción más a nuestras posibilidades terapéuticas, que tiene las obvias ventajas de ser de sencilla realización (no difiere mayormente de una esplenectomía laparoscópica), es poco invasiva, permitiendo altas precoces y, permite, en la mayoría de los casos, la preservación del bazo, como han mostrado recientes series extranjeras ${ }^{11}$.

Lo infrecuente de esta patología hace difícil reunir una casuística significativa de pacientes manejados electivamente por un abordaje laparoscópico, lo que nos invita a la colaboración de centros vasculares mediante registros o seguimiento de series multicéntricas que permitan validar esta experiencia inicial.

\section{Referencias}

1. Abbas MA, Stone WM, Fowl RJ, Gloviczki P, Oldenburg WA, Pairolero PC, et al. Splenic artery aneurysms: two decades experience at Mayo clinic. Ann Vasc Surg. 2002;16:442-9.

2. Dave SP, Reis ED, Hossain A, Taub PJ, Kerstein MD, Hollier LH. Splenic artery aneurysm in the 1990s. Ann Vasc Surg. 2000;14:223-9.

3. Berceli SA. Hepatic and splenic artery aneurysms. Semin Vasc Surg. 2005;18:196-201.

4. Trastek V, Pairolero P, Bernatz P. Splenic artery aneurysms. World J Surg. 1985;9:378-83.

5. Messina LM, Shanley CJ. Visceral artery aneurysms. Surg Clin North Am. 1997;77:425-42.

6. Wagner W, Allins A, Treiman R, Cohen JL, Foran RF, Levin PM, et al. Ruptured visceral artery aneurysms. Ann Vasc Surg. 1997;11:342-7.

7. Trastek VF, Pairolero PC, Joyce JW, Hollier LH, Bernatz PE. Splenic artery aneurysms. Surgery 1982;91:694-9.

8. De Perot M, Buhler L, Borisch B, Morel P. Management of true aneurysm of the splenic artery. Am J Surg. 1998;175:466-8.

9. Hashizume M, Ohta M, Ueno K, Okadome K, Sugimachi K. Laparoscopic ligation of splenic artery aneurysm. Surgery 1993;113:352-4.

10. Reardon PR, Otah E, Craig ES, Matthews BD, Reardon MJ. Laparoscopic resection of splenic artery aneurysms. Surg Endosc. 2005;19:488-93.

11. Pietrabissa A, Ferrari M, Berchiolli R, Morelli L, Pugliese L, Ferrari V, et al. Laparoscopic treatment of splenic artery aneurysms. J Vasc Surg. 2009;50:275-9.

12. Muscari F, Bossavy JP, Chaufour X, Ghouti L, Barret A. Laparoscopic exclusion of a splenic artery aneurysm-a case report. Vasc Endovascular Surg. 2003;37:297-300.

13. Kim BS, Do HM, Razavi M. N-butyl cyanoacrylate glue embolization of splenic artery aneurysms. J Vasc Interv Radiol. 2004;15:91-4.

14. Lupattelli T, Garaci FG, Sandhu C, Tisone G, Simonetti G. Endovascular treatment of giant splenic aneurysm that developed after liver transplantation. Transpl Int. 2003;16:756-60.

15. Tulsyan N, Kashyap VS, Greenberg RK, Sarac TP, Clair DG, Pierce G, et al. The endovascular management of visceral artery aneurysms and pseudoaneurysms. J Vasc Surg. 2007;45:276-83.

16. Saltzberg SS, Maldonado TS, Lamparello PJ, Cayne NS, Nalbandian MM, Rosen RJ, et al. Is endovascular therapy the preferred treatment for all visceral artery aneurysms? Ann Vasc Surg. 2005;19:507-15. 